Research Paper

\title{
The tri-peptide GHK-Cu complex ameliorates lipopolysaccharide- induced acute lung injury in mice
}

\author{
Jeong-Ran Park ${ }^{1,2}$, Hanbyeol Lee ${ }^{1}$, Seok-In Kim³ ${ }^{3}$ Se-Ran Yang ${ }^{1}$ \\ ${ }^{1}$ Department of Thoracic and Cardiovascular Surgery, School of Medicine, Kangwon National University, Chuncheon, Republic \\ of Korea \\ ${ }^{2}$ Institute of Medical Science, Kangwon National University, Chuncheon, Republic of Korea \\ ${ }^{3}$ Bioceltran Co., Ltd., Chuncheon, Republic of Korea \\ Correspondence to: Se-Ran Yang, email: seran.yang@gmail.com, seran@kangwon.ac.kr \\ Keywords: GHK-CU, acute lung injury, inflammation, NF-KB p65, p38 MAPK
}

Received: April 28, $2016 \quad$ Accepted: July 28, $2016 \quad$ Published: August 10, 2016

\section{ABSTRACT}

The tripeptide-copper complex glycyl-I-histidyl-I-lysine-Cu (II) (GHK-Cu) is involved in wound healing and tissue remodeling. Although GHK-Cu exhibits anti-aging and tissue renewing properties, its roles in acute lung injury (ALI)/acute respiratory distress syndrome (ARDS) are still unknown. Therefore, we examined the effects of GHK-Cu in lipopolysaccharide (LPS)-induced RAW 264.7 macrophages in vitro and ALI in mice in vivo. GHK-Cu treatment reduced reactive oxygen species (ROS) production, increased superoxide dismutase (SOD) activity while decreased TNF-a and IL-6 production through the suppression of NF-KB p65 and p38 MAPK signaling in vitro and in vivo model of ALI. Moreover, GHK-Cu attenuated LPS-induced lung histological alterations, suppressed the infiltration of inflammatory cells into the lung parenchyma in LPS-induced ALI in mice. Taken together, these findings demonstrate that GHK-Cu possesses a protective effect in LPS-induced ALI by inhibiting excessive inflammatory responses; accordingly it may represent a novel therapeutic approach for ALI/ARDS.

\section{INTRODUCTION}

Acute lung injury (ALI), along with its most severe form, acute respiratory distress syndrome (ARDS), is a disorder of acute inflammation and tissue injury that is characterized by the loss of alveolar-capillary membrane integrity, excessive transepithelial neutrophil migration, and the release of pro-inflammatory and cytotoxic mediators $[1,2]$. The incidence of ALI/ARDS ranges from 1.5 to 100 per 100,000 person-years, depending on various factors, including methodology, geography, definition, age, and associated clinical disorders [3]. It has been reported that long after lung injury, survivors have a significantly lower quality of life due to the burden of the injury and sequelae with pulmonary dysfunction [4]. ALI is mainly caused by direct lung injuries, such as the inhalation of toxic substances, and indirect systemic diseases, including sepsis, bacterial pneumonia, and severe trauma [5]. Despite advances in supportive care for survivors and improvements in anti-inflammatory interventions and treatments that inhibit the inflammatory process in lungs, the morbidity and mortality of ALI remains higher than acceptable [6].

Lipopolysaccharide (LPS, also termed endotoxin) is the major component of the outer membrane of gram-negative bacteria. It is recognized by the immune system as a marker for bacterial pathogen invasion and is responsible for the development of inflammatory responses. LPS could activate airway epithelial cells, neutrophils, and alveolar macrophages, resulting in the release of numerous inflammatory mediators, such as reactive oxygen species (ROS), tumor necrosis factor- $\alpha$ (TNF- $\alpha$ ), and interleukin-6 (IL-6).

Among existing signaling pathways, the mitogenactivated protein kinase (MAPK) and nuclear factor$\kappa \mathrm{B}(\mathrm{NF}-\kappa \mathrm{B})$ signaling pathways are key regulators of inflammatory processes. Several studies have shown that $\mathrm{NF}-\kappa \mathrm{B}-d e p e n d e n t$ gene expression plays an important role in inflammatory responses and increases the expression of genes encoding cytokines $[7,8]$. The mitogen-activated 
protein kinases (MAPKs) family, including extracellular signal-regulated kinase 1/2 (ERK1/2), p38 MAPK, and c-Jun NH2-terminal kinase (JNK1/2), has been shown to play a significant role in the mediation of signals triggered by cytokines, growth factors, and environmental stress and is involved in various cellular functions $[9,10]$. In particular, p38 MAPK is activated by various pro-inflammatory and stress stimuli and thus plays an essential role in cellular responses, including inflammation, cell proliferation, and apoptosis. Furthermore, the role of p38 MAPK in such diseases has been examined, and its inhibitors are potential therapeutic agents for their treatment $[11,12]$.

Numerous studies have reported that inflammation may directly and indirectly affect lung endothelia and pulmonary hemodynamics by activating inflammatory cells, such as neutrophils, macrophages, and lymphocytes, and proinflammatory mediators [13]. Macrophages are generally an important component in immune defense. A role of macrophages in initiating and maintaining pulmonary inflammation in lung infection or injury has been convincingly demonstrated. Recent reports suggest that lung macrophages are important orchestrators of the termination and resolution of inflammation and initiate parenchymal repair processes that are essential for a return to homeostasis with normal gas exchange [14, 15]. Furthermore, it has been hypothesized that inhibiting IL-6 and TNF- $\alpha$ production in macrophages can serve as the basis for the development of anti-inflammatory drugs.

Oxidative damage due to inflammatory responses is a major cause of lung injury during ALI [16]. Furthermore, proinflammatory mediators, such as ROS, NO, and Cox-2, play a key role in the pathogenesis of many acute inflammatory diseases [17]. These responses may enable the identification of molecules that modulate lung inflammation in ALI/ARDS.

The human tripeptide Glycyl-1-histidyl-1-lysine (GHK) is present in the plasma, saliva, and urine and is used for wound treatment and skin care [18]. It is naturally occurring, nontoxic, and readily forms complexes with copper, regulating its metabolism and improving its bioavailability. GHK tripeptide and its copper (II)-chelated form $(\mathrm{GHK}-\mathrm{Cu})$ accelerate the process of regeneration, wound healing, and antioxidant and anti-inflammatory actions [19, 20]. GHK-Cu suppresses inflammation by lowering the level of acute-phase inflammatory cytokines, including TGF- $\beta$ and TNF- $\alpha$, and reduces oxidative damage by modulating iron levels [21, 22]. Recent studies have demonstrated an antioxidant and anti-inflammatory role of $\mathrm{GHK}-\mathrm{Cu}$ to ameliorate skin damage; however, the effects of GHK-Cu on LPS-induced ALI are unknown. Therefore, we investigated whether GHK-Cu administration can attenuate lung injury via the suppression of inflammatory responses in a model of LPSinduced ALI. Furthermore, we determined the mechanism underlying the beneficial effects, including the roles of the $\mathrm{NF}-\kappa \mathrm{B}$ and MAPK pathways and oxidative enzymes.

\section{RESULTS}

\section{GHK-Cu decreased ROS production and improved SOD activity in LPS-induced RAW 264.7 cells activation}

According to a previous study, GHK-Cu can increase the level of antioxidant enzymes and SOD activity, supposedly by supplying copper, which is necessary for its function [23]. Macrophages play an important role in inflammatory events and promote the transcription of pro-inflammatory mediators. Therefore, we examined the effects of GHK-Cu on LPS-induced ROS production and SOD activity in RAW 264.7 cells. Cellular ROS was measured using DCFDA and flow cytometry. RAW 264.7 cells were treated with LPS with or without pretreatment with various concentrations of $\mathrm{GHK}-\mathrm{Cu}$. GHK-Cu did not affect cell proliferation any of the concertation examined (Supplementary Figure S1A). As shown in Figure 1A, the fluorescein derivative DCF was oxidized 59\% more in LPStreated cells compared to the control. However, pretreatment with GHK-Cu at 1,5 , and $10 \mu \mathrm{M}$ significantly decreased ROS production. SOD is an antioxidant enzyme and is markedly decreased in LPS-induced ALI [24]. We determined SOD activity after treatment with LPS with or without pretreatment with various concentrations of GHK-Cu. SOD activity was significantly decreased by approximately $19 \%$ in the LPStreated cells compared to the control (Figure 1B). In contrast, SOD activity in cells pretreated with $\mathrm{GHK}-\mathrm{Cu}(1,5$, and 10 $\mu \mathrm{M})$ improved to nearly control levels. We also determined NO secretion, is known as regulator of inflammatory response, after LPS treatment with or without $\mathrm{GHK}-\mathrm{Cu}$ pretreatment. In the present work, GHK-Cu did not change NO secretion (Supplementary Figure S1B).

\section{GHK-Cu attenuated the release of pro-inflammatory cytokines in LPS-induced RAW 264.7 cells activation}

To determine whether GHK-Cu reduces the release of inflammatory cytokines, the levels of TNF- $\alpha$ and IL-6 were confirmed by ELISA. Exposure of RAW 264.7 cells to LPS for $4 \mathrm{~h}$ significantly increased IL- 6 and TNF- $\alpha$ secretion in the culture medium to $613.2 \pm 35.1$ and 1556.3 $\pm 23.3 \mathrm{pg} / \mathrm{ml}$, respectively. The cells that were pretreated with $10 \mu \mathrm{M}$ GHK-Cu significantly decreased secretion of these molecules (Figure 2).

\section{GHK-Cu blocked the LPS-induced nuclear translocation of NF- $\kappa \mathrm{B}$ p65 and phosphorylation of NF-кB p65 in LPS-induced RAW 264.7 cells activation}

NF- $\kappa \mathrm{B}$ plays an important role in the LPS-induced expression of pro-inflammatory proteins. To investigate the molecular mechanisms of the GHK-Cu-mediated inhibition 


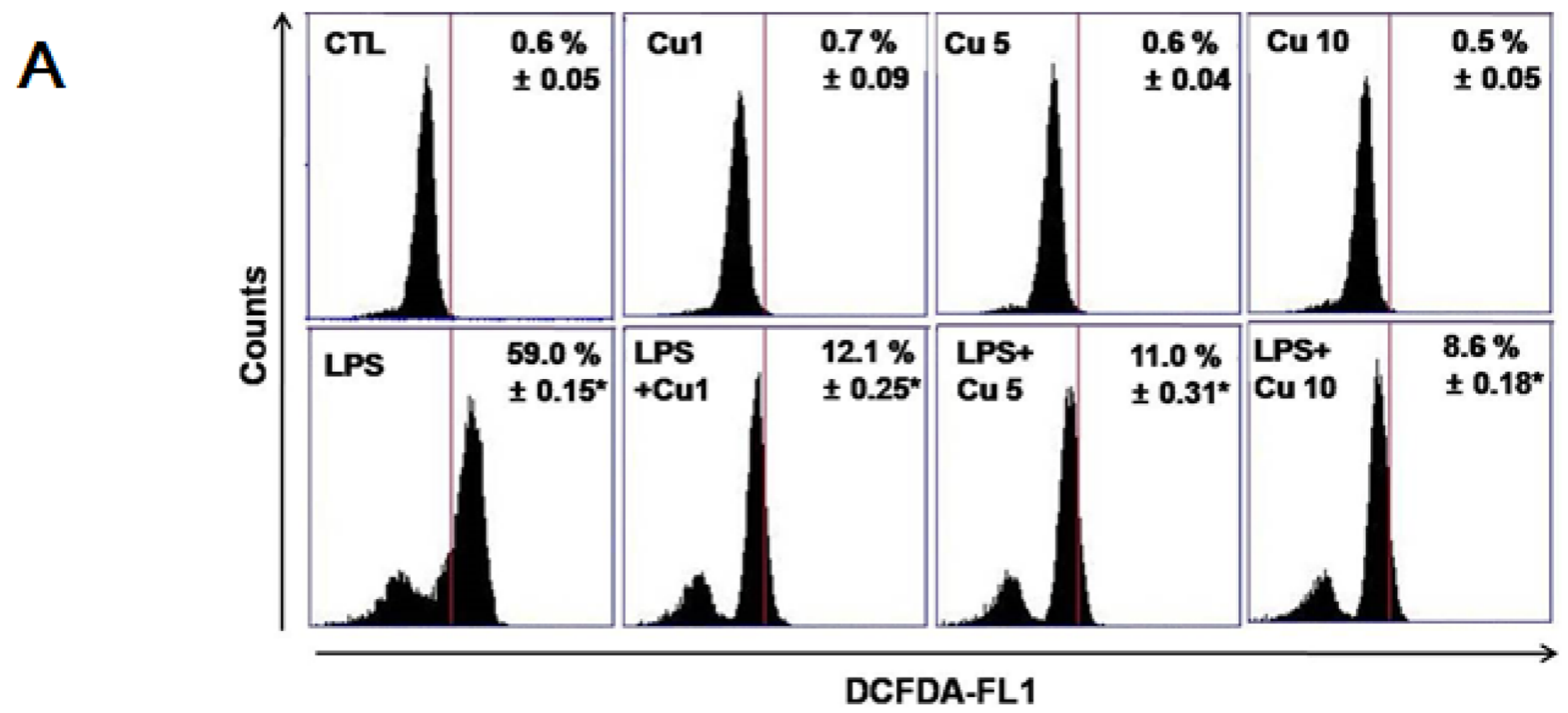

B

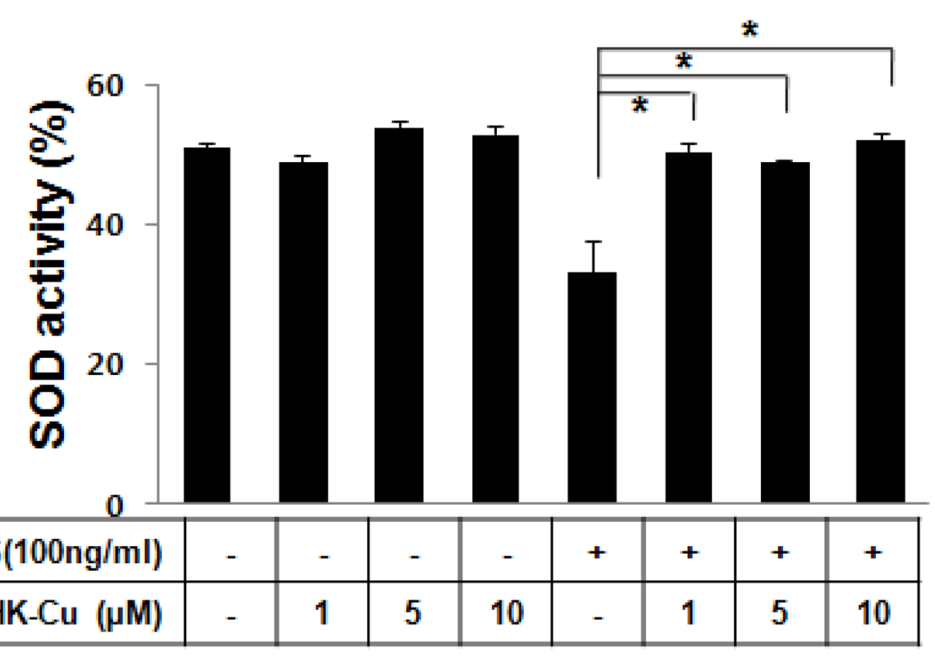

Figure 1: GHK-Cu decreased ROS production and increased SOD activity in LPS-induced RAW 264.7 cells activation. Four hours after LPS treatment with or without GHK-Cu pretreatment for $18 \mathrm{~h}$, the cells were harvested and assayed. A. The x-axis represent the intensity of intracellular DCFDA fluorescence, and the $y$-axis indicate the mean number of cells. B. Total intracellular SOD activity levels were detected using a colorimetric method. The results are representative of triplicate data. The values are presented as the means $\pm \mathrm{SD}(\mathrm{n}=3) .{ }^{*} \mathrm{p}<0.05$, statistically significant difference. CTL, control.
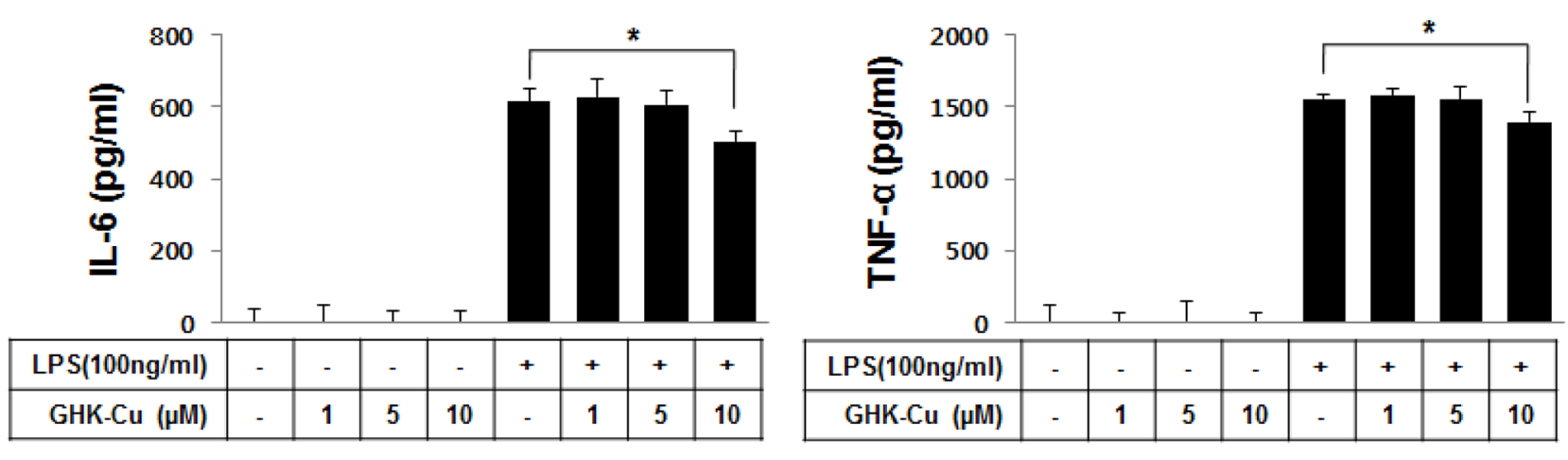

Figure 2: GHK-Cu downregulated the release of TNF- $\alpha$ and IL-6 in LPS-induced RAW 264.7 cells activation. TNF- $\alpha$ and IL-6 levels in culture supernatants were determined by ELISA. These data showed that cells that were pretreated with $10 \mu \mathrm{M} \mathrm{GHK-Cu}$ exhibited significantly decreased secretion of TNF- $\alpha$ and IL-6. The data represent the means $\pm \operatorname{SD}(n=3)$. ${ }^{*} p<0.05$, statistically significant difference. CTL, control. 
of inflammatory mediators, we examined the effects of $\mathrm{GHK}-\mathrm{Cu}$ on the activation of NF- $\mathrm{BB}$. The transactivation potential of $\mathrm{NF}-\kappa \mathrm{B}$ is increased by the phosphorylation of the NF-kB p65 subunit [25]. We examined Ser536 phosphorylation of NF- $\mathrm{B}$ p 65 by western blotting. Figure $3 \mathrm{~A}$ shows that LPS markedly induced the phosphorylation of NF-kB p65 at Ser536 in RAW 264.7 cells, while the treatment with $\mathrm{GHK}-\mathrm{Cu}(1,5$, and $10 \mu \mathrm{M})$ inhibited the LPS-stimulated phosphorylation of NF-kB p65 at Ser536. In addition, the nuclear translocation of NF- $\kappa \mathrm{B}$ was subjected to an analysis of immunofluorescence. LPS stimulation for $4 \mathrm{~h}$ caused the translocation of NF- $\mathrm{KB}$ p 65 to the nucleus in RAW 264.7 cells (Figure 3B). However, GHK-Cu treatment effectively blocked the LPS-induced nuclear translocation of NF- $\kappa$ B p65 in LPS-treated RAW 264.7 cells (Figure 3B). These results suggested that GHK-Cu could suppress the LPS-induced activation of NF- $\kappa \mathrm{B}$ signaling.

\section{GHK-Cu suppressed the activation of $\mathrm{p38}$ MAPK signaling pathway in LPS-induced RAW \\ 264.7 cells activation}

A number of studies have demonstrated that the MAPK pathway plays important roles in lung inflammatory injury. Therefore, we examined the effect of $\mathrm{GHK}-\mathrm{Cu}$ on the LPS-stimulated phosphorylation of upstream kinases, including ERK1/2, JNK1/2, and p38 MAPK, in RAW 264.7 cells. As shown in Figure 4, GHK-Cu significantly inhibited the LPS-induced phosphorylation of p38 MAPK and slightly decreased phosphorylation of JNK $1 / 2$ whereas the phosphorylation of ERK1/2 was not affected. These results suggest that the anti-inflammatory effect of GHK-Cu might result from its modulation of the p38 MAPK signaling pathway.

\section{GHK-Cu attenuated lung damage in LPS- induced ALI in mice}

Mice were administered LPS with or without GHK$\mathrm{Cu}$ pretreatment (Figure 5A). As shown in Figure 5B-5F, characteristic morphological changes were observed in lung sections after LPS administration, including notable immune cell infiltration, interstitial edema, alveolar wall thickness, and hemorrhage. GHK-Cu treatment did not affect histological changes and level of inflammatory cytokines compared to the control group (Figure 5B and 5C). Also, no toxicity was found after the $\mathrm{GHK}-\mathrm{Cu}$ treatment. The morphological changes in the lung tissue of mice with ALI suggested that the ALI model was successfully triggered under the present experimental conditions. However, in the group that received pretreatment with GHK-Cu, the pathological changes in lung tissues were markedly
A

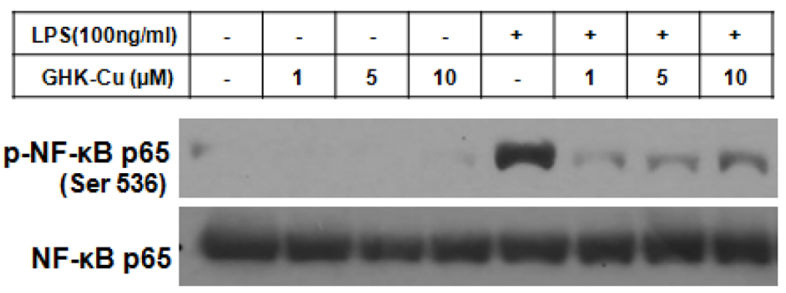

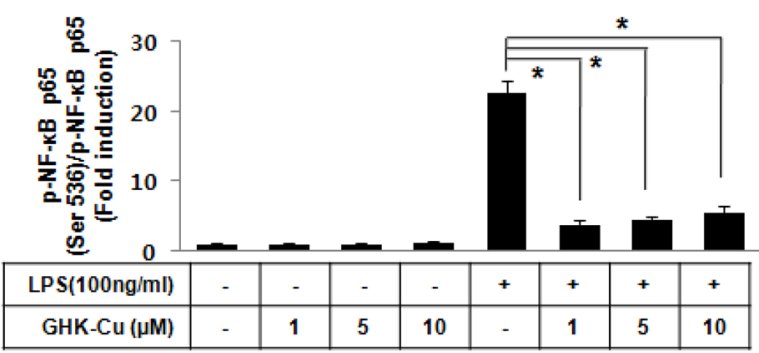

B

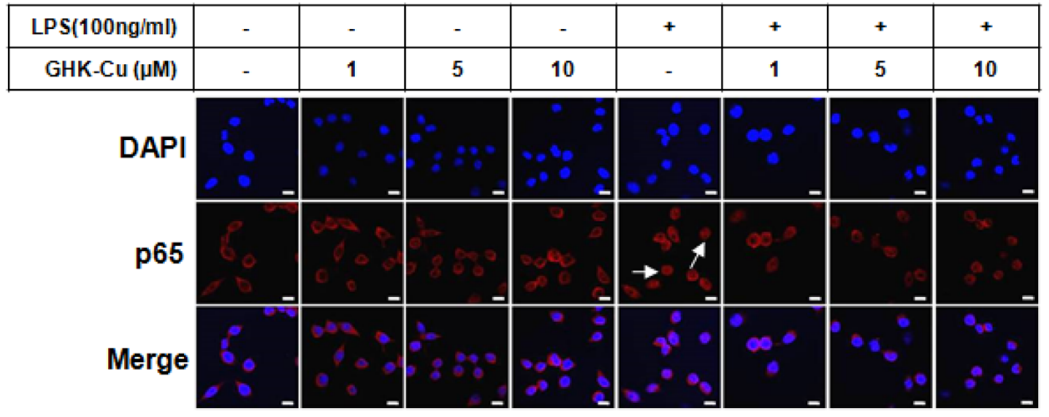

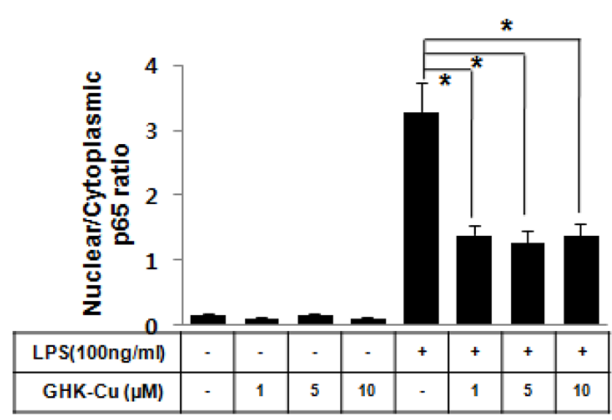

Figure 3: GHK-Cu blocked LPS-induced nuclear translocation of NF- $\mathrm{B}$ p65 and phosphorylation of NF- $\mathrm{B}$ p65 in LPS-induced RAW 264.7 cells activation. A. Phosphorylation of p65 at Ser536 was assessed by western blotting of total cell lysates with a phosphorylation site-specific antibody. The values are the means \pm SD after normalization based on total NF- $\mathrm{kB}$ p65 ( $\mathrm{n}=$ 3). A representative blot is shown for each condition. The blots were subjected to a densitometric analysis and relative quantification. $\mathbf{B}$. Immunofluorescence staining of NF- $\kappa \mathrm{B}$ p 65 and quantification of nuclear: cytoplasmic ratios of NF- $\kappa \mathrm{B}$ p65 staining. A representative $\mathrm{NF}-\kappa \mathrm{B}$ p65 stain for these conditions is shown. White arrows indicate the localization of NF- $\kappa \mathrm{B}$ p65 in the nucleus. The data represent the means \pm SD from an analysis of three separate high-power-field images. ${ }^{*} \mathrm{p}<0.05$, statistically significant difference. CTL, control. 
A

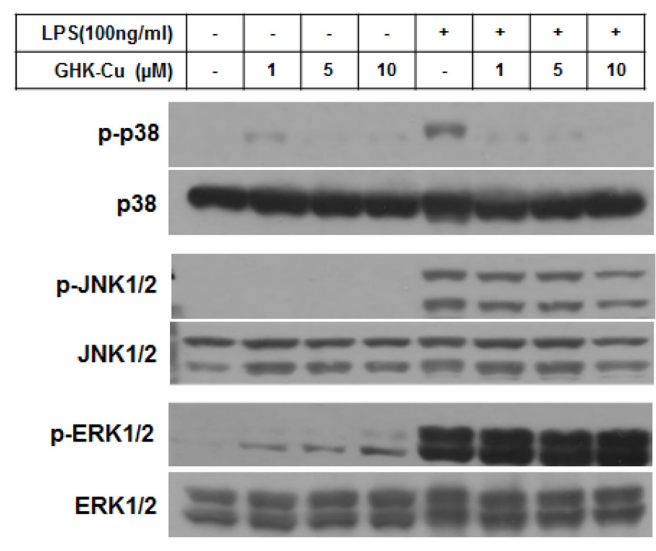

B

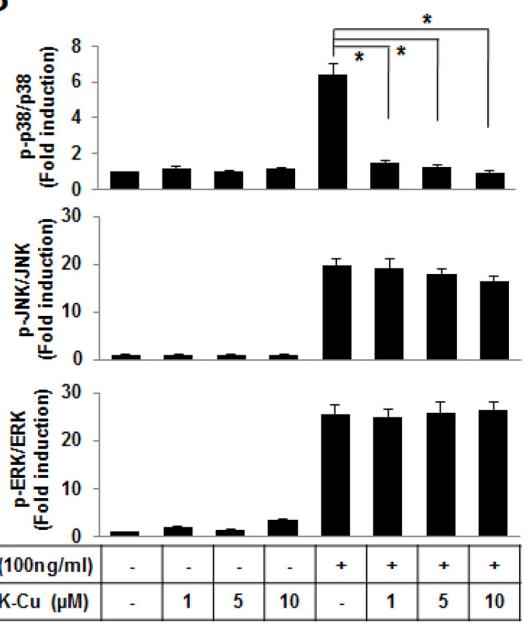

Figure 4: GHK-Cu suppressed the activation of the p38 MAPK signaling pathway in LPS-induced RAW 264.7 cells activation. A. Western blotting was performed using a specific antibody for the detection of the phosphorylation of p38 MAPK, JNK1/2, and ERK1/2. p38 MAPK, JNK1/2, and ERK1/2 were used as loading controls. B. The blots were subjected to a densitometric analysis and relative quantification. The values are the means $\pm \mathrm{SD}$ after normalization for $\mathrm{p} 38 \mathrm{MAPK}, \mathrm{JNK} 1 / 2$, and ERK1/2 $(\mathrm{n}=3)$. A representative blot is shown for each condition. ${ }^{*} \mathrm{p}<0.05$, statistically significant difference. CTL, control.
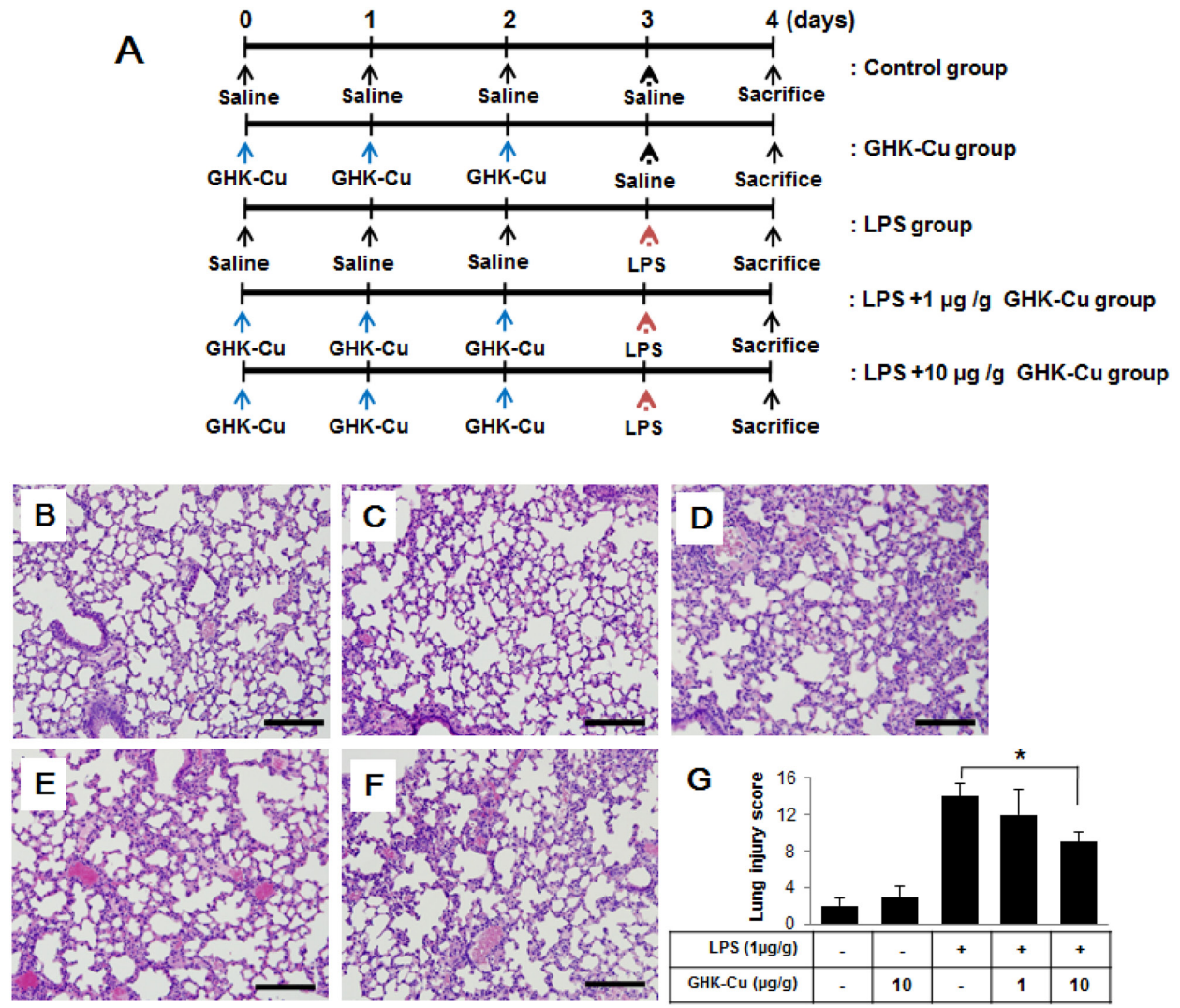

Figure 5: GHK-Cu attenuated LPS-induced acute pulmonary inflammation in mice. Mice were injected i.p. with 1 or 10 $\mu \mathrm{g} / \mathrm{g}$ GHK-Cu every $24 \mathrm{~h}$ for 3 days in $100 \mu \mathrm{l}$ of saline, while control mice were injected i.p. with $100 \mu \mathrm{l}$ of saline. Intratracheal LPS at $1 \mu \mathrm{g} / \mathrm{g}$ dissolved in $50 \mu \mathrm{l}$ of saline or saline alone was administered $24 \mathrm{~h}$ after the third injection of GHK-Cu or saline. At $24 \mathrm{~h}$ after LPS administration, the mice were sacrificed and their left lungs were fixed. Then, tissue sections were stained with hematoxylin and eosin (H \& E). A. Experimental scheme for LPS treatment with or without GHK-Cu. ( $=7$ mice per group). B. CTL; C.10 $\mu \mathrm{g} / \mathrm{g} \mathrm{GHK-Cu;} \mathrm{D.}$ LPS; E. LPS $+1 \mu \mathrm{g} / \mathrm{g}$ GHK-Cu; F. LPS $+10 \mu \mathrm{g} / \mathrm{g}$ GHK-Cu. G. Lung injury scores were calculated according to the sum of the levels of cell infiltration and damage levels as assessed from the lung sections. The data represent the means $\pm \mathrm{SD}$ ( $\mathrm{n}=7$ per group). * $\mathrm{p}<0.05$, statistically significant difference. Scale bars represent $100 \mu \mathrm{m}$. CTL, control. 
mitigated, as evidenced by the significantly reduced lung injury score (Figure 5G). These results indicated that GHK$\mathrm{Cu}$ had a protective effect on LPS-induced ALI.

\section{GHK-Cu increased antioxidant enzymes and decreased pro-inflammatory cytokines in LPS-induced ALI in mice}

We investigated whether GHK-Cu can increase antioxidant enzymes and decrease pro-inflammatory cytokines in LPS-induced ALI in mice. As summarized in Figure 6A and $6 \mathrm{~B}$, only groups administered LPS exhibited decreased SOD activity and total GSH; however, the LPS + $10 \mu \mathrm{g} / \mathrm{g}$
GHK-Cu group exhibited significantly increased SOD activity and GSH compared to the LPS alone group. Proinflammatory cytokines in the BALF were measured by an ELISA at $24 \mathrm{~h}$ after LPS administration with or without GHK-Cu pretreatment. The concentrations of TNF- $\alpha$ and IL- 6 cytokines in the BALF for the group administered only LPS were seen only with the highest individually. However, the response was markedly decreased by GHK$\mathrm{Cu}$ in the LPS $+10 \mu \mathrm{g} / \mathrm{g} \mathrm{GHK}-\mathrm{Cu}$ group (Figure $6 \mathrm{C}$ and 6D). These results demonstrated that GHK-Cu increased antioxidant enzymes and reduced the expression of proinflammatory cytokines, which in turn alleviated lung damage caused by LPS-induced ALI.

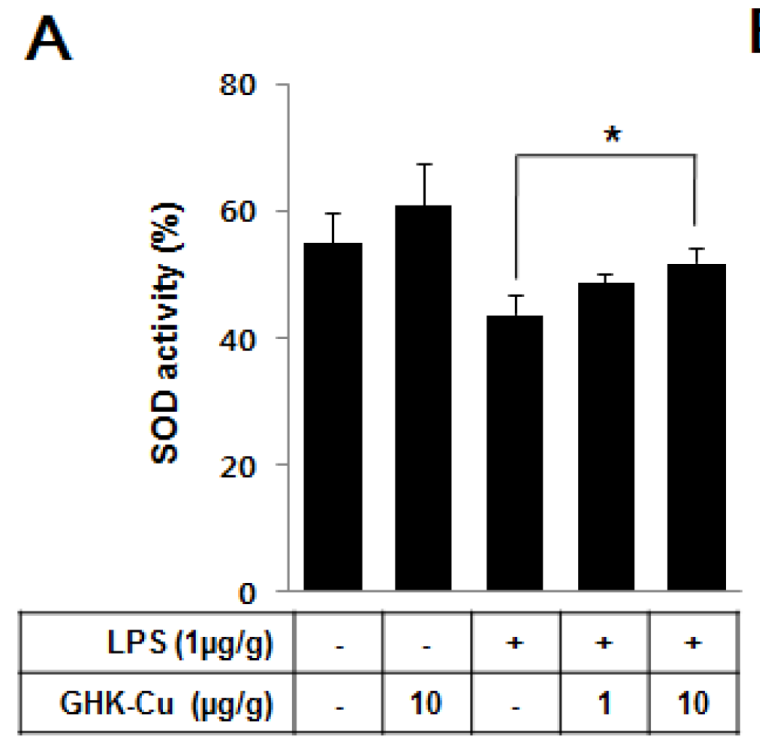

B
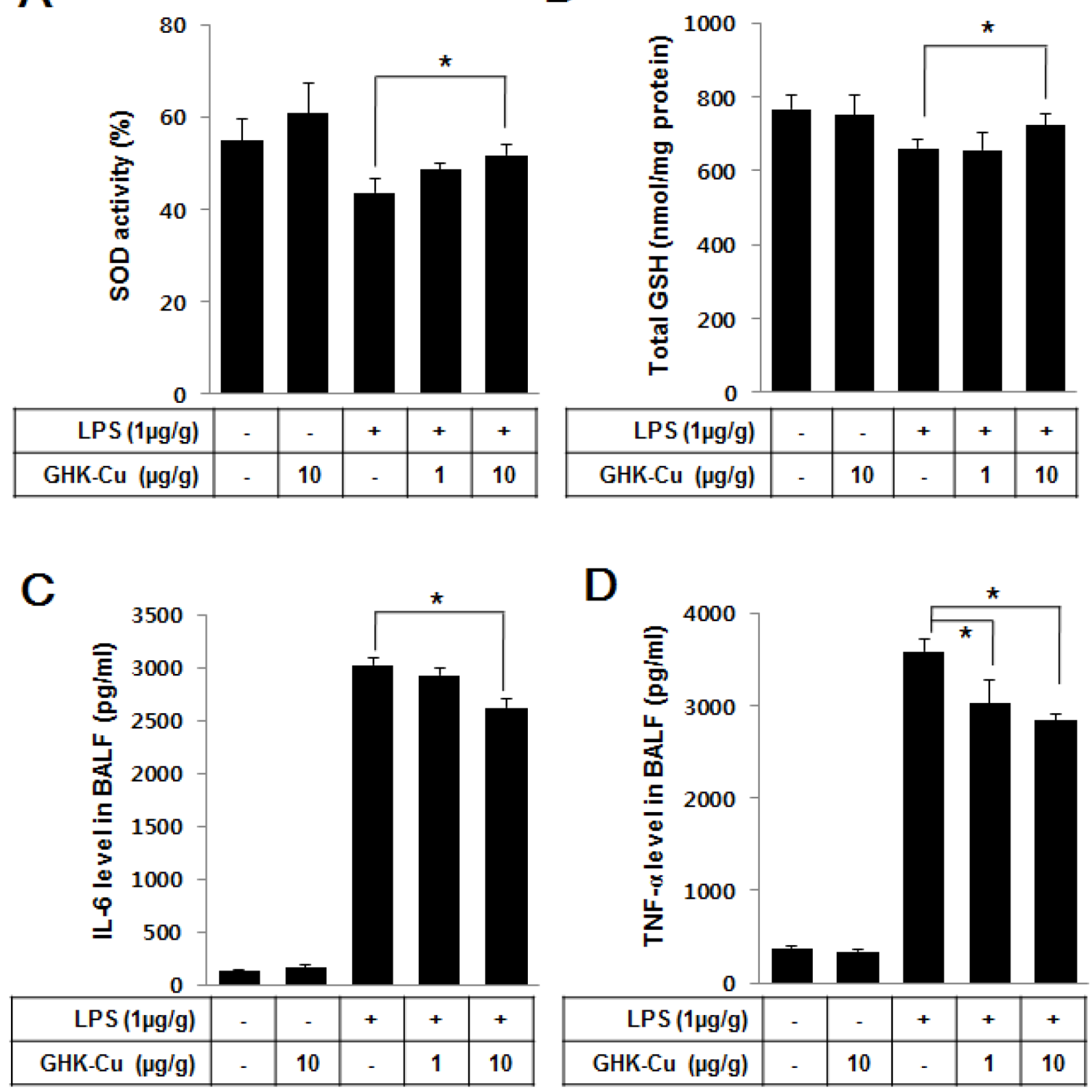

Figure 6: GHK-Cu increased antioxidant enzymes and decreased pro-inflammatory cytokines in LPS-induced ALI in mice. At $24 \mathrm{~h}$ after LPS administration, the mice were sacrificed, and lung homogenates and BALF were prepared. A. Total intracellular SOD activity levels were detected using a colorimetric method. B. The level of glutathione (GSH) was measured in the lung homogenate. C. IL-6 and D. TNF- $\alpha$ were detected by ELISA. The data represent the mean $\pm \operatorname{SD}(n=3) .{ }^{*} p<0.05$, statistically significant difference. 


\section{GHK-Cu reduced immune cell infiltration and alveolar permeability in the BALF}

Activated neutrophils have been linked to the production of oxidative stress, which is a critical factor in the pathophysiology of ALI. To further identify the antiinflammatory properties of $\mathrm{GHK}-\mathrm{Cu}$, it is important to calculate immune cell infiltration into the lungs. Therefore, we evaluated alterations in immune cell infiltration in the lungs of LPS-administered mice with or without GHK-Cu pretreatment. MPO activity is a marker of neutrophils [26]. The level of lung MPO activity (Figure 7A) and infiltrated neutrophils (Figure 7B and Supplementary Figure S3) were markedly increased in the LPS group, whereas the LPS +1 or $10 \mu \mathrm{g} / \mathrm{g}$ GHK-Cu group exhibited a trend toward lower levels in a dose-dependent manner. The total cell counts in the BALF were also lower in the LPS $+10 \mu \mathrm{g} / \mathrm{g}$ GHK-Cu group than the LPS group (Figure 7C). ALI exhibits intra-alveolar edema and increased alveolar permeability. We assessed lung permeability by measuring the total protein concentration in the BALF. The total proteins were significantly increased in the LPS-administered group, but decreased the LPS +10 $\mu \mathrm{g} / \mathrm{g}$ GHK-Cu group in the BALF (Figure 7D).

GHK-Cu suppressed the phosphorylation of $\mathrm{p38}$ MAPK, JNK1/2 and NF-KB p65 in LPS-induced ALI in mice

The MAPK pathways have been demonstrated to participate in the activation of NF-kB in LPS-induced ALI.
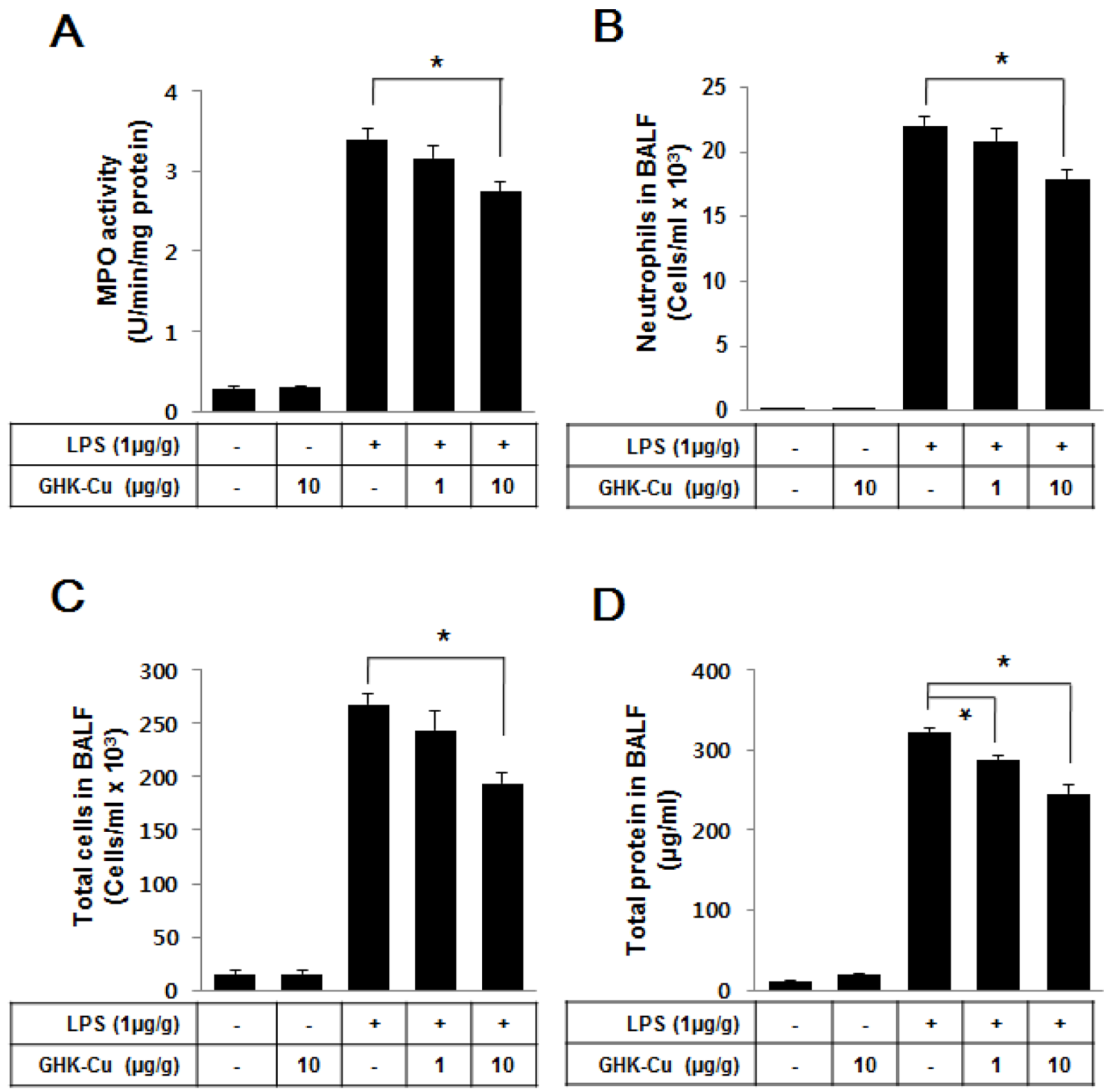

Figure 7: GHK-Cu reduced immune cell infiltration and total protein in BALF. At $24 \mathrm{~h}$ after LPS administration, the mice were sacrificed and lung BALF was prepared. Cells in the BALF were collected and cytospin preparations were made. A. Myeloperoxidase (MPO) activity, B. neutrophils counts, $\mathbf{C}$. total cell counts and $\mathbf{D}$. total proteins were determined as described in Materials and Methods. The data represent the mean $\pm \mathrm{SD}(\mathrm{n}=3) . * \mathrm{p}<0.05$, statistically significant difference. 
As shown in Figure 8, the results from Western blotting showed that GHK-Cu had little effect on LPS-induced phosphorylation of ERK1/2, however, it significantly reduced the phosphorylation of p38 MAPK and JNK1/2 in LPS $+10 \mu \mathrm{g} / \mathrm{g}$ GHK-Cu group compared to the LPS group. In parallel with phosphorylation of MAPK, the effect of GHK-Cu on phosphorylation of NF- $\mathrm{kB}$ p 65 were measured by Western blotting. The phosphorylation of NF- $\kappa B$ p65 at Ser536 in lung increased significantly after LPS administration compared with control group, while the treatment with GHK-Cu reduced phosphorylation of NF-кB p65 at Ser536 in LPS $+10 \mu \mathrm{g} / \mathrm{g}$ GHK-Cu group compared to the LPS group (Figure 8).

\section{DISCUSSION}

Copper peptides are naturally occurring small protein fragments that possess high affinity to copper ions in human serum and cerebrospinal fluid (PMID21898044). GHK-Cu accelerates wound healing, improves the success of transplanted skin, and has anti-inflammatory effects on the skin $[19,20]$. It has a physiological role in antiinflammatory responses and tissue remodeling. In this present study, we demonstrated that GHK-Cu exerts a protective effect in LPS-induced ALI.
Oxidative stress refers to elevated intracellular levels of ROS. High level of ROS, which that lead to cellular damage, are critical for LPS-induced inflammation [27]. Accordingly, we first performed experiments to determine the effects of GHK-Cu on intracellular ROS production. The pretreatment of cells with GHK-Cu significantly reduced LPS-induced ROS production. Few studies have examined the relationship between GHK-Cu and ROS [28]. Under normal physiological conditions, oxidative stress is improved by anti-oxidative enzymes acquired through SOD, catalase, and GSH. Notably, superoxide anions related to oxidative damage are converted to hydrogen peroxide by SOD, which is then metabolized to water via catalase or GSH [29]. In this study, we demonstrated that pretreatment with GHK-Cu increased SOD activity and total GSH in LPS-induced ALI. These observations suggest that GHK-Cu is capable of reducing serious lung damage by improving anti-oxidant enzymes.

ALI, an inflammatory disorder, is characterized, among other events, by the production of significant amounts of free radicals and nitrogen reactive species as well as inflammatory cytokines, such as TNF- $\alpha$ and IL-6. TNF- $\alpha$ is a pivotal proinflammatory cytokine and is regarded as an endogenous mediator. Excessive production of cytokines can be induced by inflammatory stimuli, such as LPS in macrophages, and it increases the immune response,
A

\begin{tabular}{|c|c|c|c|c|c|c|c|c|c|c|}
\hline LPS(1ug/g) & - & - & - & - & + & + & + & + & + & + \\
\hline GHK-Cu $(\mu \mathrm{M})$ & - & - & 10 & 10 & - & - & 1 & 1 & 10 & 10 \\
\hline \multicolumn{11}{|c|}{ 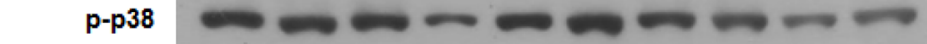 } \\
\hline \multicolumn{11}{|l|}{ p38 } \\
\hline p-JNK1/2 & $=$ & $=$ & $\longrightarrow$ & - & $=$ & 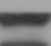 & $=$ & $=$ & $=$ & $\overline{-1}$ \\
\hline JNK1/2 & - & 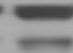 & - & 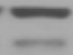 & $=$ & & $=$ & $=$ & $=$ & $=$ \\
\hline p-ERK1/2 & E & 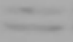 & $\equiv$ & $=$ & $=$ & $=$ & $=$ & $=$ & $=$ & $=$ \\
\hline ERK1/2 & 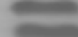 & & 4 & $\mathrm{sen}$ & $\mathrm{s}=\mathrm{n}$ & & $1=$ & & & \\
\hline $\begin{array}{r}\text { p-NF-kB p65 } \\
\text { (Ser 536) }\end{array}$ & $=$ & $=$ & $=$ & $=$ & ש & 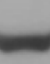 & $=$ & $\omega$ & $\longrightarrow$ & $\longrightarrow$ \\
\hline NF-кB p65 & & & & & & & & & 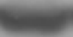 & 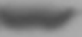 \\
\hline
\end{tabular}

B

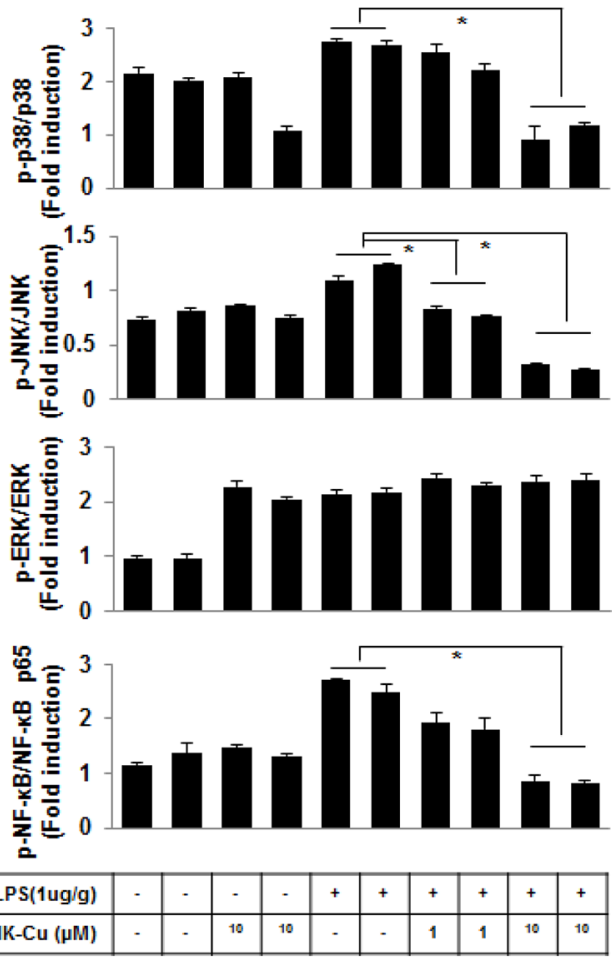

Figure 8: MAPKs and NF- $\kappa B$ signaling pathway is involved in the anti-inflammatory effect of GHK-Cu. A. Phosphorylation of NF- $\mathrm{BB}$ p 65 at Ser536, p38 MAPK, ERK1/2, and JNK1/2 was assessed by western blotting of lung homogenates with a phosphorylation site-specific antibody. NF-KB p65 and p38 MAPK, JNK1/2, and ERK1/2 were used as loading controls. B. The blots were subjected to a densitometric analysis and relative quantification. The values are the means $\pm \mathrm{SD}$ after normalization for NF- $\mathrm{B}$ p65, p38 MAPK, JNK1/2, and ERK1/2 $(n=3)$. A representative blot is shown for each condition. $* \mathrm{p}<0.05$, statistically significant difference. 
which in turn results in inflammation [30]. Therefore, the inhibition of pro-inflammatory cytokines is a target for antiinflammatory therapies. Our results showed that $\mathrm{GHK}-\mathrm{Cu}$ could significantly suppress TNF- $\alpha$ and IL-6 expression in vitro and in vivo, suggesting that GHK-Cu is useful for the development of novel anti-inflammatory therapies.

The transcriptional regulator $\mathrm{NF}-\kappa \mathrm{B}$ plays a pivotal role in inflammation owing to its ability to induce the transcription of an array of inflammatory genes, especially those involved in the regulation of pro-inflammatory molecules [31]. Nuclear translocation of NF- $\kappa B$ p65 induced target gene activation. NF- $\kappa \mathrm{B}$ p65 activation is associated with alveolar macrophage activity, which is the major source of inflammatory cytokines [32]. Our results demonstrate that $\mathrm{GHK}-\mathrm{Cu}$ has the ability to inhibit the LPS-induced phosphorylation of NF- $\kappa$ B p65 at Ser536, an event associated with NF- $\kappa \mathrm{B}$ activation, as well as the nuclear translocation of NF- $\kappa \mathrm{B}$ p65 in macrophages. The therapeutic potential of inhibiting the NF- $\kappa \mathrm{B}$ pathway in chronic inflammatory diseases and inflammatory bowel diseases has also been reported [33]. These findings concur with our finding that the transcriptional inhibition of proinflammatory mediators by $\mathrm{GHK}-\mathrm{Cu}$ is associated with the blockade of the NF- $\mathrm{B}$ signaling pathway.

In addition to NF- $\kappa \mathrm{B}$, LPS is a potent activator of the MAPK pathway, which regulates cytokine release in macrophages [34]. We explored whether the $\mathrm{GHK}-\mathrm{Cu}$ has an effect on the activation of p38 MAPK, ERK1/2, and JNK1/2 signaling molecules in LPS-induced RAW 264.7 macrophages in vitro and ALI in mice in vivo. Our results showed that p38 MAPK, ERK1/2, and JNK1/2 activation occurred in LPS-induced ALI. However, GHK$\mathrm{Cu}$ pretreatment markedly suppressed the LPS-induced phosphorylation of p38 MAPK, had little effect of JNK1/2, but not ERK1/2. Furthermore, we also examined the level of pro-inflammatory cytokines and phosphorylation of p38 MAPK and NF-kB to further investigate whether $\mathrm{GHK}-\mathrm{Cu}$ can reduce anti-inflammatory response on the peritoneal macrophages. As expected, GHK-Cu decreased LPS-induced TNF- $\alpha$ and IL-6 secretion, and suppressed phosphorylation of p38 MAPK and NF$\kappa \mathrm{B}$ p65 (Supplementary Figure S2). N-acetylcysteine (NAC), a ROS scavenger, has been evaluated in various experimental conditions including ALI. Hsu et al., reported that NAC decreased p38 MAPK activity to the basal level as well as moderated LPS-induced inflammatory cytokine levels [35]. These results suggested that the suppression of p38 MAPK phosphorylation by $\mathrm{GHK}-\mathrm{Cu}$ is involved in the inhibition of the LPS-induced production of proinflammatory mediators in LPS-induced ALI.

ALI is characterized by excessive neutrophil infiltration into the lung, and neutrophils serve as a therapeutic target to attenuate inflammation in ALI. Although neutrophil activation is vital for host defense, excessive activation leads to tissue damage by cytotoxic and immune cell-activating agents, such as proteinases, cytokines, and ROS [36]. Therefore, to determine whether GHK-Cu affects neutrophil infiltration to alleviate ALI, we analyzed neutrophil infiltration into the lung by detecting total MPO, an enzyme released from activated neutrophils, and the neutrophil count in lung BALF. These results suggested that GHK-Cu can mitigate neutrophil infiltration into the lung during LPS-induced ALI.

To the best of our knowledge, this is the first study to investigate the protective effect of $\mathrm{GHK}-\mathrm{Cu}$ via its antiinflammatory and anti-oxidant properties and its possible mechanisms in LPS-induced ALI (Figure 9). Our results

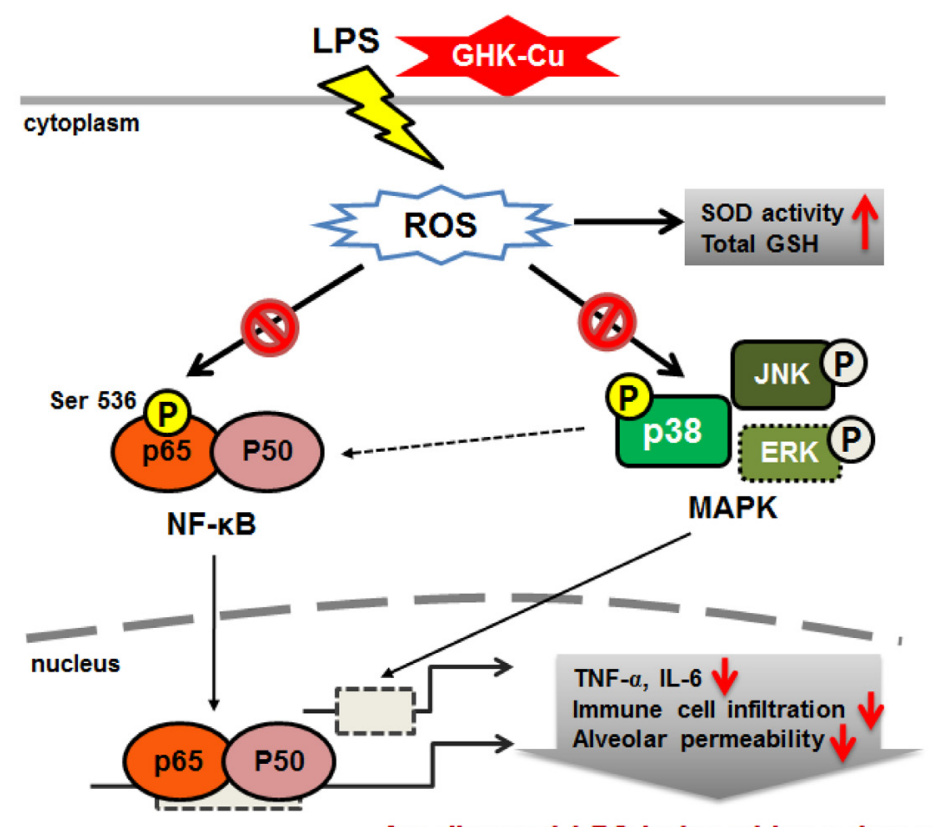

Ameliorated LPS-induced lung damage

Figure 9: Schematic diagram showing the proposed mechanisms underlying the attenuation of LPS-induced inflammatory response by GHK-Cu. 
provide valuable insight into the molecular actions of GHK-Cu and revealed its underlying mechanisms. These findings indicated that $\mathrm{GHK}-\mathrm{Cu}$ are a promising agent for the clinical prevention and therapy of inflammatory disease.

\section{MATERIALS AND METHODS}

\section{Materials}

GHK-Cu (MW 340.38; soluble in water) was provided by Bioceltran Corporation Ltd., (Chuncheon, Korea). Lipopolysaccharide (Escherichia coli O111:B4), thioclycollate and 2',7'-dichlorofluorescin-diacetate (DCFDA) were purchased from Sigma (MO, USA). Antibodies against phospho-ERK1/2 (\#4370), total ERK1/2 (\#4695), phospho-p38 MAPK (\#4511), total p38 MAPK (\#9212), phospho-JNK1/2 (\#4668), total JNK1/2 (\#9252), phospho-NF-кB p65 (Ser536, \#3033) and $\beta$-actin (\#3700) were purchased from Cell Signaling (MA, USA). Antibodies against NF- $\kappa \mathrm{B}$ p65 (sc-372) was purchased from Santa Cruz Biotechnology (CA, USA).

\section{Cell cultures, preparation of peritoneal macrophages, cell proliferation and cell stimulation}

The murine macrophage cell line RAW 264.7 cells were purchased from Korea Cell Line Bank (KCLB, Seoul, Korea). Peritoneal macrophages were harvested 4 days after the i.p. injection of 3\% thioglycollate. Macrophages were washed and plated in 24 -well plates at $0.5 \times 10^{6}$ cells per well. After incubation for $2 \mathrm{~h}$ at $37^{\circ} \mathrm{C}$, the wells were washed 3 times to remove all nonadherent cells. Finally, the culture medium was replaced. Cells were grown in RPMI 1640 medium containing $10 \%$ fetal bovine serum (Hyclone, USA) in a $37^{\circ} \mathrm{C}$ humidified incubator containing $5 \% \mathrm{CO}_{2}$ and $95 \%$ air. Cells were pretreated without or with $\mathrm{GHK}-\mathrm{Cu}$ at various concentrations for $18 \mathrm{~h}$. The cells were then stimulated with LPS (100 ng/ $\mathrm{ml}$ ) for indicated time period. The culture supernatants or cells were collected for the following analysis. Cell proliferation was assessed using 3-(4,5-dimethylthiazol2-yl)-2,5-diphenyltetra-zolium bromide (MTT; SigmaAldrich) assay.

\section{Measurement of intracellular ROS (DCFDA)}

The level of intracellular ROS was detected using cell permeable fluorescent probe 2', 7'-dichlorofluorescindiacetate (DCFDA). Intracellular detection of ROS was achieved by incubating cells with $10 \mu \mathrm{M}$ CM-DCFDA for $30 \mathrm{~min}$ at $37^{\circ} \mathrm{C}$ prior to LPS and GHK-Cu treatment. Cells were then detached by gentle scraping with a cell scraper and analyzed by Accuri-C6 flow cytometry using FL1 channel (BD biosciences, USA).

\section{Immunostaining}

Cells were seeded on round glass cover slips in 24well plates $24 \mathrm{~h}$ prior to treatment with GHK-Cu and LPS. After LPS treatment, the samples were washed with cold PBS and fixed in $4 \%$ paraformaldehyde in PBS for $15 \mathrm{~min}$ at room temperature. The cells were then permeabilized with $0.2 \%$ Triton $\mathrm{X}-100$ in PBS for $10 \mathrm{~min}$ at room temperature and incubated overnight at $4^{\circ} \mathrm{C}$ with either anti- NF- $\kappa$ B p65 antibody. After 3 washes with PBS, the cells were incubated with Alexa Fluor 546-conjugated goat-anti rabbit IgG (Invitrogen, CA, USA) for $1 \mathrm{~h}$ at room temperature and then washed again with PBS. The images were captured using a confocal laser-scanning microscope.

\section{Western blot analysis}

Whole cell lysates and lung homogenates were prepared with RIPA lysis buffer containing with a protease inhibitor cocktail (GenDEPOT; P3100, P3200). The protein concentrations in the lysates were determined by Bicinchoninic (BCA) Protein Assay Kit (Thermo Scientific, IL, USA: 23225), following the manufacturer's instructions. Then, the $20 \mathrm{ug}$ of isolated soluble proteins was mixed with $5 X$ SDS-PAGE sample buffer (Tech\&Innovation, Chuncheon, Korea; BSS9005), electrophoresed in a $10 \%$ polyacrylamide gel and transferred to nitrocellulose transfer membrane $0.45 \mathrm{~mm}$ (BIO-RAD, CA, USA; 162-0115). The membranes were blocked with $5 \%$ skim milk (dilution in TBS with 0.05 $\%$ Tween 20 (TBST)) and incubated with primary antibodies $\left(1: 1000\right.$, dilution in TBST) for overnight at $4^{\circ} \mathrm{C}$. After sufficiently washed with TBST, the membranes were incubated with polyclonal anti-rabbit/mouse HRP-conjugated secondary antibodies for $1 \mathrm{~h}$ at room temperature. After that, the membranes were washed with TBST and detected using an ECL detection solution.

\section{Animals}

Thirty five male C57BL/6 mice, 10 weeks old, were purchased form Doo Yeol Biotech (Seoul, Korea). Animals were housed in the Animal Facility of Kangwon National University with an environmentally controlled room $\left(23^{\circ} \mathrm{C}\right.$ $\pm 2,55 \% \pm 5 \%$ relative humidity) with $12 \mathrm{~h}$ dark-light cycles. During the experiment, the animals were fed with a standard laboratory chow and water. All experiments were approved and followed the regulations of the Institutional Animal Care and Use Committee (IACUC, KW-130503-1, Kangwon National University, Korea).

\section{Model of LPS-induced acute lung injury}

ALI was induced by intratracheal administration of $1 \mu \mathrm{g} / \mathrm{g}$ LPS in $50 \mu \mathrm{l}$ of saline. With this model, ALI, as characterized by neutrophils infiltration into the lung 
interstitium and airways, development of interstitial edema, and increased pulmonary proinflammatory cytokine production, occurs after injection of LPS, with the greatest degree of injury being present $24 \mathrm{~h}$ after LPS exposure [37]. Briefly, mice were anesthetized with Zoletil 50 (Virbac, France; $30 \mathrm{mg} / \mathrm{kg}$, i.p.). The tongue was then gently extended, and the LPS solution was deposited into the pharynx [38]. Thirty five male C57BL/6 mice were randomly divided into 5 groups $(n=5)$ : control group, LPS group, GHK-Cu group (10 $\mu \mathrm{g} / \mathrm{g})$, low dosage group (1 $\mu \mathrm{g} / \mathrm{g}$ GHK-Cu + LPS $)$, and high dosage group $(10 \mu \mathrm{g} / \mathrm{g}$ $\mathrm{GHK}-\mathrm{Cu}+\mathrm{LPS})$. For the GHK-Cu pretreatment groups, mice were injected intraperitoneally with $\mathrm{GHK}-\mathrm{Cu}$ (1 or $10 \mu \mathrm{g} / \mathrm{g}$, every $24 \mathrm{~h}$, for 3 days) dissolved in saline before intratracheal administration of LPS. The mice in the control group were administrated with saline instead. $24 \mathrm{~h}$ later, mice were sacrificed. There were no deaths associated with this model for GHK-Cu and/or LPS administration.

\section{Histopathological analysis}

To examine the histological examination, the left lung lobes were trimmed and fixed by immersion in $10 \%$ neutral formalin for $24 \mathrm{~h}$. Paraffin-embedded lungs were cut into $4 \mu \mathrm{m}$ thick sections and subsequently stained with hematoxylin and eosin for histological analysis. Lung injury was graded from 0 (normal) to 4 (severe) in four categories. Lung injury score was evaluated according to the sum of the damage level such as the neutrophil infiltration, congestion, edema and alveolar wall thickness. [39].

\section{Harvest of lungs and bronchoalveolar lavage fluid (BALF)}

Lungs were harvested $24 \mathrm{~h}$ after LPS administration. BALF was collected from mice to analyze the cell counts, total protein content and cytokine production. Mice were sacrificed, and their tracheas were immediately lavaged two times via a catheter with $1 \mathrm{ml}$ of ice-cold PBS. BALF collected was centrifuged at $2,000 \mathrm{rpm}$ for $5 \mathrm{~min}$ at $4^{\circ} \mathrm{C}$. The supernatants were collected and then stored at $-80^{\circ} \mathrm{C}$ until the cytokine assay and determination of total protein content. Differential cell counts were assessed on cytological preparations obtained by cytospin centrifuge (Cellspin, Hanil, Korea $)$ of $100 \mu \mathrm{l}$ of the diluted BALF $\left(1 \times 10^{6}\right.$ cells $/$ $\mathrm{mL}$ in ice-cold PBS). The slides were fixed and stained with Hema-3 stain (Thermo Fisher Scientific) according to the manufacturer's instructions, and cells were counted under a light microscope. At least 400 cells were counted for each preparation. The number of neutrophils were expressed as the absolute number from the total cell counts.

\section{Measurement of Myeloperoxidase (MPO) activity and nitrite determination}

MPO activity was measured using a minor modification of a previously described method [38]. In brief, lung tissue was homogenized in $0.5 \mathrm{ml}$ of $0.5 \%$ hexadecyltrimethyl ammonium bromide in $50 \mathrm{mM}$ potassium phosphate buffer ( $\mathrm{pH}$ 6.0). The homogenate was centrifuged at $12,000 \mathrm{rpm}$ for $15 \mathrm{~min}$ at $4^{\circ} \mathrm{C}$ and the supernatant was collected for assay MPO activity as determined by measuring the $\mathrm{H}_{2} \mathrm{O}_{2}$-dependent oxidation of $o$-dianisidine solution (3,3'-dimethoxybenzidine dihydrochloride in potassium phosphate buffer, $\mathrm{pH} 6.0$ ) at $450 \mathrm{~nm}$ using an Epoch microplate spectrophotometer (BioTek, VT, USA). NO detection in culture media was performed using Griess reagent (Sigma-Aldrich, Saint Louis, MO) at $540 \mathrm{~nm}$.

\section{Determination of SOD and GSH activity}

SOD activity was assessed using the commercial SOD determination kit (Sigma-Aldrich, Saint Louis, MO). The rate of the activity of SOD was measured the absorption spectrum about formazan form of WST-1 (2-(4-lodophenyl)-3-(4-nitrophenyl)-5-(2,4disulfophenyl)-2H-tetrazolum, monosodium salt), highly water-soluble tetrazolium salt, that generate a watersoluble formazan dye with reduction of a superoxide anion. Consequentially, amount of superoxide anion is proportional to the absorbance at $440 \mathrm{~nm}$, and means inhibition activity of SOD. The assay for quantitative determination of glutathione (GSH) levels was conducted as previously described method by Rahman et al. [40]. For the GSH assay, the lungs were harvested, washed in PBS, and then homogenated in $0.1 \mathrm{M}$ phosphate buffer containing $5 \mathrm{mM}$ EDTA, $0.1 \%$ (vol/vol) Triton X-100, and $0.6 \%$ (wt/vol) sulfosalicylic acid. The cellular debris was collected by centrifugation, the supernatant concentration were determined by BCA assay Kit. The proteins were incubated with $0.67 \mathrm{mg} / \mathrm{ml} \mathrm{DTNB}$ and 10 $\mathrm{U} / \mathrm{ml}$ glutathione reductase in $0.1 \mathrm{M}$ potassium phosphate buffer with $5 \mathrm{mM}$ EDTA disodium salt, $\mathrm{pH}$ 7.5. After $30 \mathrm{~s}, 0.67 \mathrm{mg} / \mathrm{ml}$ b-NADPH was added. The actual total GSH concentration in the samples were determined by calculated linear regression values from standard curve every $30 \mathrm{~s}$ for a total time of $2 \mathrm{~min}$ at $412 \mathrm{~nm}$ absorbance.

\section{ELISA for TNF- $\alpha$ and IL-6 cytokines}

RAW 264.7 cells $\left(5 \times 10^{5}\right.$ per well $)$ were seeded in 24-well plates containing RPMI 1640 supplemented with $10 \%$ FBS for 1 day to become nearly confluent. On the second day, the cells were pretreated with the indicated concentrations of GHK-Cu $18 \mathrm{~h}$ before treatment with LPS at $37^{\circ} \mathrm{C}$ for $4 \mathrm{~h}$. The cultured media were collected and stored at $-80^{\circ} \mathrm{C}$ for the following cytokine assay. The bronchoalveolar lavage fluid (BALF) supernatant was collected after centrifugation and stored at $-80^{\circ} \mathrm{C}$ before the cytokine assay. Immunoreactive TNF- $\alpha$ and IL-6 were measured by Duoset enzyme-linked immunosorbent assay (ELISA) development kits (R\&D systems, MN, USA) according to the manufacturer's instruction. 


\section{Statistical analysis}

All the statistical data were analyzed by GraphPad Prism 5.0 (GraphPad Software, CA, USA). The results were expressed as the mean $\pm \mathrm{SD}$. Comparisons between two groups were performed using an unpaired student's $t$-test. One-way analysis of variance (ANOVA) was used for comparisons between multiple groups. A value of $p<0.05$ was considered statistically significant.

\section{ACKNOWLEDGMENTS}

This work was supported by the National Research Foundation of Korea (NRF) grant funded by the Korea government (NRF- 2014R1A2A2A01003737).

\section{CONFLICTS OF INTEREST}

The authors declare no potential conflicts of interest.

\section{REFERENCES}

1. Hamilton KL, Franklin L, Roy S and Schrott LM. Prenatal opiate exposure attenuates LPS-induced fever in adult rats: role of interleukin-1beta. Brain Res. 2007; 1133:92-99.

2. Johnson ER and Matthay MA. Acute lung injury: epidemiology, pathogenesis, and treatment. J Aerosol Med Pulm Drug Deliv. 2010; 23:243-252.

3. Wind J, Versteegt J, Twisk J, van der Werf TS, Bindels AJ, Spijkstra JJ, Girbes AR and Groeneveld AB. Epidemiology of acute lung injury and acute respiratory distress syndrome in The Netherlands: a survey. Respiratory medicine. 2007; 101:2091-2098.

4. Rubenfeld GD, Caldwell E, Peabody E, Weaver J, Martin DP, Neff M, Stern EJ and Hudson LD. Incidence and outcomes of acute lung injury. N Engl J Med. 2005; 353:1685-1693.

5. Matuschak GM and Lechner AJ. Acute lung injury and the acute respiratory distress syndrome: pathophysiology and treatment. Mo Med. 2010; 107:252-258.

6. Geboers DG, de Beer FM, Tuip-de Boer AM, van der Poll T, Horn J, Cremer OL, Bonten MJ, Ong DS, Schultz MJ and Bos LD. Plasma suPAR as a prognostic biological marker for ICU mortality in ARDS patients. Intensive Care Med. 2015; 41:1281-1290.

7. Ma L, Zhao Y, Wang R, Chen T, Li W, Nan Y, Liu X and Jin F. 3,5,4'-Tri-O-acetylresveratrol Attenuates Lipopolysaccharide-Induced Acute Respiratory Distress Syndrome via MAPK/SIRT1 Pathway. Mediators Inflamm. 2015; 2015:143074.

8. Gao M, Chen L, Yu H, Sun Q, Kou J and Yu B. Diosgenin down-regulates NF-kappaB p65/p50 and p38MAPK pathways and attenuates acute lung injury induced by lipopolysaccharide in mice. Int Immunopharmacol. 2013; 15:240-245.
9. Reibman J, Talbot AT, Hsu Y, Ou G, Jover J, Nilsen D and Pillinger MH. Regulation of expression of granulocytemacrophage colony-stimulating factor in human bronchial epithelial cells: roles of protein kinase $\mathrm{C}$ and mitogenactivated protein kinases. J Immunol. 2000; 165:1618-1625.

10. Chu X, Ci X, Wei M, Yang X, Cao Q, Guan M, Li H, Deng Y, Feng $\mathrm{H}$ and Deng $\mathrm{X}$. Licochalcone a inhibits lipopolysaccharide-induced inflammatory response in vitro and in vivo. J Agric Food Chem. 2012; 60:3947-3954.

11. Kaminska B. MAPK signalling pathways as molecular targets for anti-inflammatory therapy--from molecular mechanisms to therapeutic benefits. Biochim Biophys Acta. 2005; 1754:253-262.

12. Yong HY, Koh MS and Moon A. The p38 MAPK inhibitors for the treatment of inflammatory diseases and cancer. Expert Opin Investig Drugs. 2009; 18:1893-1905.

13. Mafra de Lima F, Naves KT, Machado AH, Albertini R, Villaverde $\mathrm{AB}$ and Aimbire F. Lung inflammation and endothelial cell damage are decreased after treatment with phototherapy (PhT) in a model of acute lung injury induced by Escherichia coli lipopolysaccharide in the rat. Cell Biol Int. 2009; 33:1212-1221.

14. Rubins JB. Alveolar macrophages: wielding the doubleedged sword of inflammation. Am J Respir Crit Care Med. 2003; 167:103-104.

15. Marsh LM, Cakarova L, Kwapiszewska G, von Wulffen W, Herold S, Seeger W and Lohmeyer J. Surface expression of CD74 by type II alveolar epithelial cells: a potential mechanism for macrophage migration inhibitory factor-induced epithelial repair. American journal of physiology Lung cellular and molecular physiology. 2009; 296:L442-452.

16. Roth E, Manhart $\mathrm{N}$ and Wessner B. Assessing the antioxidative status in critically ill patients. Curr Opin Clin Nutr Metab Care. 2004; 7:161-168.

17. Rehman MU, Yoshihisa Y, Miyamoto Y and Shimizu T. The anti-inflammatory effects of platinum nanoparticles on the lipopolysaccharide-induced inflammatory response in RAW 264.7 macrophages. Inflamm Res. 2012; 61:1177-1185.

18. Pickart L, Vasquez-Soltero JM and Margolina A. GHK Peptide as a Natural Modulator of Multiple Cellular Pathways in Skin Regeneration. BioMed research international. 2015; 2015:648108.

19. Pickart L, Vasquez-Soltero JM and Margolina A. The human tripeptide $\mathrm{GHK}-\mathrm{Cu}$ in prevention of oxidative stress and degenerative conditions of aging: implications for cognitive health. Oxid Med Cell Longev. 2012; 2012:324832.

20. Pickart L. The human tri-peptide GHK and tissue remodeling. J Biomater Sci Polym Ed. 2008; 19:969-988.

21. Canapp SO, Jr., Farese JP, Schultz GS, Gowda S, Ishak AM, Swaim SF, Vangilder J, Lee-Ambrose L and Martin FG. The effect of topical tripeptide-copper complex on healing of ischemic open wounds. Vet Surg. 2003; 32:515-523. 
22. Miller DM, DeSilva D, Pickart L and Aust SD. Effects of glycyl-histidyl-lysyl chelated $\mathrm{Cu}$ (II) on ferritin dependent lipid peroxidation. Adv Exp Med Biol. 1990; 264:79-84.

23. Arul V, Gopinath $\mathrm{D}$, Gomathi $\mathrm{K}$ and Jayakumar $\mathrm{R}$. Biotinylated GHK peptide incorporated collagenous matrix: A novel biomaterial for dermal wound healing in rats. J Biomed Mater Res B Appl Biomater. 2005; 73:383-391.

24. Kinnula VL and Crapo JD. Superoxide dismutases in the lung and human lung diseases. Am J Respir Crit Care Med. 2003; 167:1600-1619.

25. Viatour P, Merville MP, Bours V and Chariot A. Phosphorylation of NF-kappaB and IkappaB proteins: implications in cancer and inflammation. Trends Biochem Sci. 2005; 30:43-52.

26. Abraham E. Neutrophils and acute lung injury. Crit Care Med. 2003; 31:S195-199.

27. Lum H and Roebuck KA. Oxidant stress and endothelial cell dysfunction. Am J Physiol Cell Physiol. 2001; 280:C719-741.

28. Guilloreau L, Combalbert S, Sournia-Saquet A, Mazarguil $\mathrm{H}$ and Faller P. Redox chemistry of copper-amyloid-beta: the generation of hydroxyl radical in the presence of ascorbate is linked to redox-potentials and aggregation state. Chembiochem. 2007; 8:1317-1325.

29. Annapurna A, Ansari MA and Manjunath PM. Partial role of multiple pathways in infarct size limiting effect of quercetin and rutin against cerebral ischemia-reperfusion injury in rats. Eur Rev Med Pharmacol Sci. 2013; 17:491-500.

30. Crespo A, Filla MB, Russell SW and Murphy WJ. Indirect induction of suppressor of cytokine signalling-1 in macrophages stimulated with bacterial lipopolysaccharide: partial role of autocrine/paracrine interferon-alpha/beta. Biochem J. 2000; 349:99-104.

31. Kim SH and Shin TY. Anti-inflammatory effect of leaves of Eriobotrya japonica correlating with attenuation of p38
MAPK, ERK, and NF-kappaB activation in mast cells. Toxicol In Vitro. 2009; 23:1215-1219.

32. Moraes LB, Murakami AH, Fontes B, Poggetti RS, van Rooijen N, Younes RN, Heimbecker AM and Birolini D. Gut ischemia/reperfusion induced acute lung injury is an alveolar macrophage dependent event. J Trauma. 2008; 64:1196-1200; discussion 1200-1191.

33. Yamamoto $\mathrm{Y}$ and Gaynor RB. Therapeutic potential of inhibition of the NF-kappaB pathway in the treatment of inflammation and cancer. J Clin Invest. 2001; 107:135-142.

34. Bak MJ, Truong VL, Kang HS, Jun M and Jeong WS. Antiinflammatory effect of procyanidins from wild grape (Vitis amurensis) seeds in LPS-induced RAW 264.7 cells. Oxid Med Cell Longev. 2013; 2013:409321.

35. Hsu HY and Wen MH. Lipopolysaccharide-mediated reactive oxygen species and signal transduction in the regulation of interleukin-1 gene expression. The Journal of biological chemistry. 2002; 277:22131-22139.

36. Grommes J and Soehnlein O. Contribution of neutrophils to acute lung injury. Mol Med. 2011; 17:293-307.

37. Abraham E, Arcaroli J, Carmody A, Wang H and Tracey KJ. HMG-1 as a mediator of acute lung inflammation. J Immunol. 2000; 165:2950-2954.

38. Tsuruta Y, Park YJ, Siegal GP, Liu G and Abraham E. Involvement of vitronectin in lipopolysaccaride-induced acute lung injury. J Immunol. 2007; 179:7079-7086.

39. Itoh T, Obata H, Murakami S, Hamada K, Kangawa K, Kimura $\mathrm{H}$ and Nagaya N. Adrenomedullin ameliorates lipopolysaccharide-induced acute lung injury in rats. American journal of physiology Lung cellular and molecular physiology. 2007; 293:L446-452.

40. Rahman I, Kode A and Biswas SK. Assay for quantitative determination of glutathione and glutathione disulfide levels using enzymatic recycling method. Nat Protoc. 2006; 1:3159-3165. 